\title{
Phone Use and Aging: Do Spatial Interface Metaphors Help?
}

\author{
Mark Turner and Roxane Cortes \\ Department of Psychology, University of Portsmouth, King Henry Building, \\ King Henry I Street, Portsmouth, PO1 2DY, UK \\ mark.turner@port.ac.uk, \\ roxane.cortes@myport.ac.uk
}

\begin{abstract}
This study compared the usability of two different speech activated automated telephone services with a group of older adults, aged between 50 and 87. All participants completed a series of information search tasks with a standard numbered menu system, and with a specially developed menu system designed around a spatial metaphor. Automated menu systems presented a significant barrier for older adults with only 59\% of participants able to complete all search tasks. Task completion improved when using the spatial metaphor-based version of the service. The spatial ability of participants was related to successful task completion with both services. Time taken to complete search tasks correlated with working memory capacity only in the case of the standard service and not the metaphor-based service. The incorporation of spatial metaphor within automated telephone systems may therefore provide an important aid to navigation when developing future services with older adults in mind.
\end{abstract}

Keywords: Aging, Interface Design, Automated Telephones, Spatial Metaphor.

\section{Introduction}

The proportion of older people in the UK population is growing rapidly. Population forecasts indicate that by 2033, 29\% of the UK's population will be over 60 years old (Office for National Statistics, [1]). Reductions in personal mobility and autonomy that occur with age also mean that older adults are becoming more reliant on technology for communication and continued connection with society.

The use of devices such as the mobile telephone and voice activated menu systems now form an essential part of communication within modern society in a wide variety of contexts such as banking, transport and health care; yet their use may present a considerable barrier to independent living for older adults. Older people are less likely to adopt new technologies (Renaud \& Van Biljon, [2]), perform less well when navigating through phone-based menu systems (Ziefle \& Bay, [3]) and experience more difficulties understanding synthetic speech (Roring, Hines \& Charness, [4]).

Age related declines in cognitive abilities are also well documented; older adults have been repeatedly shown to perform worse than younger adults on laboratory tasks 
involving memory and controlled temporal processing (e.g Kausler, Salthouse, \& Saults [5]); such skills are commonly required when interacting with serially presented auditory information via telephone. A more effective means of presenting information through automated telephone menu systems is therefore required to support the domestic needs and continued inclusion of older adults in society.

Previous research by the applicant with young adults has demonstrated that voiceactivated telephone systems based around the use of a spatial interface metaphor are effective at improving caller performance compared to standard telephone menu systems (Howell, Love \& Turner, [6]). The speed of learning and acceptability of voice-activated menu interfaces are also related to the extent to which callers report visualising the phone service they are using, a task which becomes easier when spatial metaphor is used (Howell, Love \& Turner, [7]). Logan, Sanders, Synder, Morris \& Buckner, [8] showed that providing the elderly with appropriate processing strategies to aid recall on a memory task can be an effective way of partially offsetting agerelated memory deficits. It follows that the provision of an effective metaphor for interacting with a phone based menu system may present a viable means of improving the accessibility of such telephone systems for older adults.

\subsection{Aims}

The aim of the current study was to examine whether a voice-activated telephone menu system designed around a spatial metaphor could be used to enhance the usability of telephone services for older adults, when factors such as cognitive ability and visual memory are taken into account.

Specifically, the study addresses:

1. Whether a spatial-metaphor based service would be easier and faster to use than a standard service that uses numbered menus.

2. Whether ability to visualize the structure of each service improves users' interactions with the service.

3. Whether individual differences in cognitive attributes such as working memory capacity and spatial ability, and previous experience of telecommunication systems can be used to predict success at using automated services.

\section{Method}

\subsection{Design}

The study used a 2 (trial) x 2 (service type) repeated measures design. A hierarchically structured phone menu system was used which consisted of five levels of service messages and prompts, with a maximum of three menu options to choose from at each level. Users completed two search tasks with each version of the service which required them to successfully retrieve up to four pieces of information before exiting the service.

The non-metaphor based version of the service was designed in the same style as commonly available automated phone services by pairing numbers with menu options (e.g. 'Say ' 1 ' for Arts and Entertainment, say '2' for Eating out, etc.). 
The office filing system service required the caller to select one of three filing cabinets and then to navigate through different drawers, partitions and folders to the information required. Commands within the system were designed to encourage spatial processing of the system structure (e.g. top/bottom, front/middle/rear, etc.). In addition, participants were provided with a cue card depicting three filing cabinets which provided a simple visual representation of the top level of the service, designed to facilitate visualization (Fig. 1).
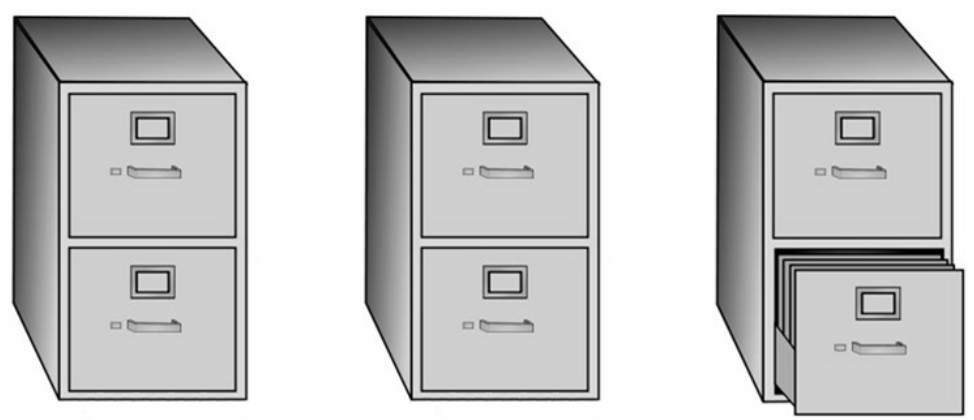

Fig. 1. Cue card for metaphor-based service structure

\subsection{Measures}

The following measures were gathered from all participants in the study:

Task Performance Measures. Two objective measures of task performance were collected during each participant's interaction with the service; successful task completion and the time taken to complete each task. The total time taken to complete each task was logged in seconds from the beginning of the first 'level 1' dialogue to the end of the 'exit' dialogue.

Service Usability. A 20-item post-exposure usability questionnaire was used to evaluate subjective attitudes towards each implementation of the service, balanced for positively and negatively worded items. Items (e.g. "I sometimes felt lost when using the service") were responded to using a 7-point Likert scale, where higher scores indicated greater difficulty with using the service. In addition, participants were asked to indicate 'yes' or 'no' whether they had been able to visualize each service.

Technographic Questionnaire. This consisted of a series of 18 questions designed to assess each person's previous experience of using mobile telephones, automated telephone services and other relevant forms of technology. Items (e.g. "How often do you use a mobile telephone to access automated services such as banking or cinema information?") were responded to using a visual analogue scale with scores in the range 0 to 10, where higher scores indicated greater frequency of use.

Spatial Ability. The AH4 Group Test of General Intelligence (Heim, [9]) Part II was used to assess spatial ability. This is a two-section questionnaire consisting of 12 practice items and 65 test items in each section. Participants are presented with a 
target diagrammatic stimulus or series of stimuli and are required to perform visual analogies, subtractions or superimpositions in order to correctly identify an appropriate transformation of the original target. Participants are permitted a maximum of 10 minutes to complete Part II, resulting in a numerical indication of spatial ability between 0 and 65 .

Working Memory Span Test. This test was used to assess participants working memory capacity derived from Baddeley [10]. Participants were serially presented with sentences which were either semantically correct or incorrect. Sentences were read aloud to participants. Each sentence consisted of 5 words (e.g. "The boy brushed his teeth"). After listening to each sentence the participant was asked to say 'yes' if the sentence made sense, or 'no' if the sentence did not make sense. After each block of 5 sentences, participants were asked to recall the last word of each of the 5 sentences in that block. Eight blocks of sentences were presented in total, yielding a maximum working memory score of 40 correctly recalled items.

\subsection{Participants}

Twenty-two individuals took part in the study consisting of 12 males and 10 females. The age of participants ranged from 50 to 87 years $(M=61.0$ years, $S D=10.9)$ with participants being recruited from members of a local old people's group and older working members of a local organization. The study was conducted in accordance with the British Psychological Society (BPS) Ethical Principles for Conducting Research with Human Participants as defined by the BPS Code of Ethics and Conduct [11] and was approved by the relevant Research Ethics Committee of our organization. All participants gave their consent to take part in this research and for their data to be used in this report.

\subsection{Procedure}

Each participant was tested individually within a 1-hour session. Firstly participants completed the technographic questionnaire, followed by the working memory span test and AH4 assessment of spatial ability. Each participant then completed one practice task and two recorded search tasks each with the standard service and the metaphor-based service respectively. A 'Wizard of Oz' methodology was used for the experiment which involved the experimenter simulating the functionality of a fully automated system to create the illusion that the user was interacting with a real voiceactivated telephone service. Participants gave verbal rather than keyed responses and listened to the service prompts via a headset. Both services were based around the provision of information for a mock telephone city guide service (Howell et al, [6]). Each task required participants to find and retrieve specific information from within the guide (e.g. "Find the names of 2 weekend art exhibitions and then exit the service"). Task completion times were recorded using a stopwatch. On completion of the tasks within each service, participants completed the usability questionnaire. 


\section{Results}

\subsection{Task Performance Measures}

Task Completion. Use of the automated telephone services presented difficulties for the current sample with only 13 out of the 22 participants able to successfully complete all tasks with standard service $(59.1 \%)$ but significantly more $(77.3 \%)$ were able to complete all tasks with metaphor-based service $\left.\chi^{2}(1, \mathrm{n}=22)=9.35, \mathrm{p}=0.005\right)$.

Fig. 2 shows the mean task completion rate achieved by participants on the first and second trail with each service as a percentage of the total number of pieces of information that were to be retrieved.

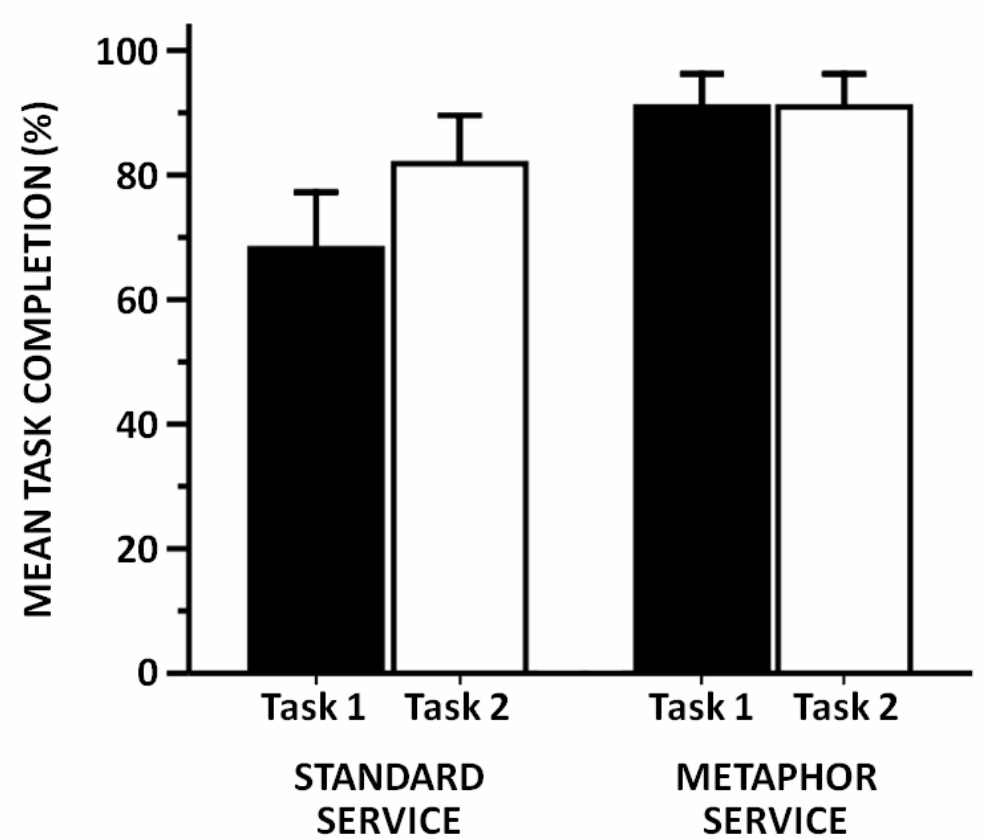

Fig. 2. Mean (+1 SE) successful task completion (percent correct information retrieved) for two calls made to a standard service and metaphor-based phone service by 22 older adults

A 2x2 (service $\mathrm{x}$ trial) repeated measures analysis of variance ANOVA revealed a main effect of metaphor $\left(\mathrm{F}(1,21)=8.871, \mathrm{p}=0.007 ; \eta^{2}=0.297\right)$. Successful task completion was greatest for the metaphor service $(90.9 \%, \mathrm{SD}=25.1 \%)$ and lower for standard service $(68.2 \%, \mathrm{SD}=42.4 \%)$ on task 1 increasing to $81.8 \%, \mathrm{SD}=36.3 \%)$ on task 2. Bonferroni Pairwise comparisons revealed a significant difference to occur between the metaphor and standard service on task 1, suggesting that information could be better retrieved from the metaphor service on first use. No main effect of 
task $\left(\mathrm{F}(1,21)=2.278, \mathrm{p}=0.110 \mathrm{~ns} ; \eta^{2}=0.117\right)$ and no interaction effect $(\mathrm{F}(1,21)=2.278$, $\left.\mathrm{p}=0.110 \mathrm{~ns} ; \eta^{2}=0.117\right)$ were found suggesting that the difference in task completion rates between the two services remained broadly consistent across both tasks.

Task Duration. For the 13 participants who successfully completed all trials (Fig. 3), the completion times did not increase significantly from the first call to second call $\left(\mathrm{F}(1,12)=1.85, \mathrm{p}=0.198 \mathrm{~ns} ; \eta^{2}=0.134\right)$. There was also no overall difference $\left(\mathrm{F}(1,12)=0.121, \mathrm{p}=0.734 \mathrm{~ns} ; \eta^{2}=0.10\right)$ in the time taken to use standard service $(\mathrm{M}=2.7$ minutes; $\mathrm{SD}=1.0)$ and metaphor service $(\mathrm{M}=2.8$ minutes; $\mathrm{SD}=1.1)$. A significant interaction was found though between service type and trial $\left(\mathrm{F}(1,12)=10.941, \mathrm{p}=0.006 ; \eta^{2}=0.477\right)$ which arose from the fact that less time was taken to use the standard service ( $M=2.6$ minutes) than metaphor service $(M=3.0$ minutes) during performance of the second task (Bonferroni Pairwise comparison, $\mathrm{p}=0.015)$. This difference may have been an artifact of the specific information that participants were asked to seek which was varied across services to prevent rehearsal.

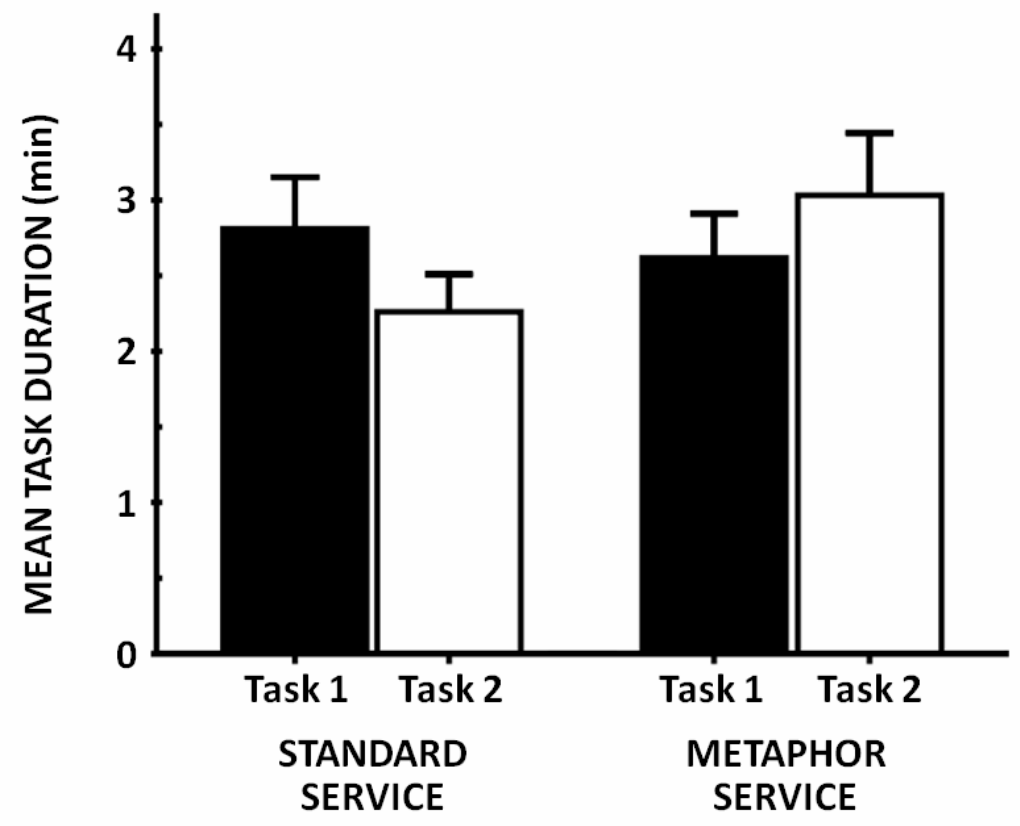

Fig. 3. Mean (+1 SE) task completion duration (minutes per call) for successful calls made to a standard service and metaphor-based phone service by 13 older adults

\subsection{Service Usability and Visualization}

The self-reported usability of the two telephone service indicated participants found both services difficult to use, with no overall difference $(\mathrm{t}(21)=1.42, \mathrm{p}=0.16 \mathrm{~ns}$; $\mathrm{d}=0.43$ ) being found between the subjective evaluations of the standard service $(\mathrm{M}=3.91, \mathrm{SD}=1.33)$ and the metaphor-based service $(\mathrm{M}=4.41, \mathrm{SD}=1.02)$. 
A greater number of participants reported being able to visualize the metaphorbased service $(86.4 \%)$ than were able to visualize the standard service $(68.2 \%)$. Visualization was also related to service usability. Participants who reported being able to visualize the standard service found this service significantly easier to use $(\mathrm{M}=4.32 ; \mathrm{SD}=1.00)$ than those who could not visualize the service $(\mathrm{M}=3.04 ; \mathrm{SD}=1.6)$ $(\mathrm{t}(20)=2.30, \mathrm{p}=0.032 ; \mathrm{d}=0.98)$. However, in the case of the metaphor-based service the difference in usability ratings between those who could $(\mathrm{M}=4.52, \mathrm{SD}=1.00)$ and could not $(\mathrm{M}=3.75, \mathrm{SD}=1.05)$ visualize the service was not found to be statistically significant $(\mathrm{t}(20)=1.23, \mathrm{p}=0.23 \mathrm{~ns} ; \mathrm{d}=0.74)$.

\subsection{Individual Difference Variables}

Table 1 shows Pearson correlation coefficients between the two objective measures of task performance for both versions of the phone system with individual participant attributes including spatial ability and working memory capacity.

Table 1. Pearson ( $\mathrm{r}$ ) Correlation Coefficients $(* \mathrm{p}<0.05 * * \mathrm{p}<0.01 ; 20 \mathrm{df})$ between cognitive ability measures for 22 older adults and task performance measures when using the standard and metaphor-based services

\begin{tabular}{lllll}
\hline Attribute & \multicolumn{2}{c}{ Standard Service } & \multicolumn{2}{c}{ Metaphor-based Service } \\
& $\begin{array}{c}\text { Task } \\
\text { Completion }\end{array}$ & $\begin{array}{c}\text { Task } \\
\text { Duration }\end{array}$ & $\begin{array}{c}\text { Task } \\
\text { Completion }\end{array}$ & $\begin{array}{c}\text { Task } \\
\text { Duration }\end{array}$ \\
\hline Age & $-0.56^{* *}$ & -0.06 & $-0.67^{* *}$ & -0.01 \\
Spatial Ability (AH4) & $0.67^{* *}$ & $-0.62^{* *}$ & $0.61^{* *}$ & -0.33 \\
Working Memory Span & $0.54^{*}$ & $-0.59^{* *}$ & 0.32 & -0.20 \\
Service Usability & $0.47^{*}$ & $-0.50^{*}$ & -0.29 & 0.06 \\
Technographic experience & 0.15 & -0.05 & -0.06 & -0.17 \\
\hline
\end{tabular}

These analyses suggest that successful task completion declined significantly with increased participant age for both the standard service $(\mathrm{r}(20)=-0.56, \mathrm{p}<0.01)$ and the metaphor-based service $(\mathrm{r}(20)=-0.67 \mathrm{p}<0.01)$. However, the time taken to complete tasks was unrelated to age for both services.

Participants with higher spatial ability scores were found to have better task completion rates for both the standard service $(\mathrm{r}(20)=0.67, \mathrm{p}<0.01)$ and metaphorbased service $(r(20)=0.61, p<0.01)$. Those with greater spatial ability also completed tasks more quickly with the standard service $(r(20)=-0.62 \mathrm{p}<0.01)$ although no significant relationship was found between spatial ability and speed of completion for the metaphor-based service $(\mathrm{r}(20)=-0.33$, ns).

Working memory capacity was related to task performance for the standard service where by those with a greater working memory span for phonological information were able to complete tasks more quickly $(\mathrm{r}(20)=-0.59 \mathrm{p}<0.01)$ and more accurately $(\mathrm{r}(20)=0.54 \mathrm{p}<0.05)$ with the standard service. However, working memory span was not related to the speed or accuracy of task performance when using the metaphorbased service. 
Subjective ratings of service usability were also associated with quicker $(\mathrm{r}(20)=-$ $0.50 \mathrm{p}<0.05)$ and more successful task completion rates $(\mathrm{r}(20)=0.47 \mathrm{p}<0.05)$, but again this trend was only observed for the standard service and not for the metaphorbased service.

Participants' speed and accuracy of task performance was found to be unrelated to their previous experience of using automated telephone systems in the case of both the standard and metaphor-based phone services.

\section{Discussion}

Of the two telephone systems examined, the interface which used a spatial metaphor based around an office filing system was easier to visualize and resulted in more successful service use among older adults than a standard numbered menu system. The requirement to complete only 2 tasks with each menu system following only one practice trial was designed to mimic the actual context in which such systems would be used, where participants will often have little prior knowledge or exposure to menu options or structure. Under these conditions, when examining initial task performance with each system, successful task completion was found to be significantly better for the metaphor-based service. This result is consistent with previous research that suggests visual navigational aids can improve the usability of menu structures for older adults (Ziefle \& Bay, 2006).

Contrary to expectations, the metaphor-based service did not result in faster task completion times than the standard service. Whilst the absolute time taken to complete tasks provides a valid indicator of the level of involvement with each service required by the user, it does not provide a true test of usability when comparing the two services directly since service prompts were of different lengths in each system. Since the metaphor-based service tended to have longer service prompts but did not produce significantly longer task completion times overall, this may provide a strong indication of the potential utility of this service. Numbered menu systems are well established in contemporary automated phone services. It could therefore be seen as encouraging that a novel system design with longer service prompts was not evaluated by older adults as being less preferred or indeed did not result in a significant increase in the time taken to use the service use overall.

One possible explanation for the superior user performance with the metaphorbased system is assumed to be the emphasis this service places on the use of spatial as opposed to verbal cues when interacting with the service. Those with greater spatial ability were found to perform more successfully on tasks with both services, which may indicate that the ability to imagine and manipulate the menu service structure within visual memory provides the key to better performance. Fewer participants reported being able to visualize the standard service structure and performance with the standard service only was found to be related to working memory span. This may indicate that in the absence of a reliable visual representation of a telephone system, service users are more reliant on phonological aspects of memory when searching serially presented verbal information, and that providing a suitable visual structure to 
represent phone systems to users may help alleviate the burden placed on the phonological loop component of working memory that is experienced by older adults when interacting with such systems.

Metaphor-based interfaces may therefore provide an important aid to automated service use for older adults and present a possibility for future system design. Further research might give consideration to the utility of alternative metaphors to support service structures beyond an office-based system that might benefit older adults or offer a broader, more universal design.

Acknowledgments. This research was supported by a grant from the British Psychological Society Undergraduate Research Assistantship Scheme.

\section{References}

1. Office for National Statistics. Population Estimates: UK population grows to 61.8 million (2009), http://www.statistics.gov.uk/cci/nugget.asp?id=6 (retrieved February 2, 2010)

2. Renaud, K., Van Biljon, J.: Predicting Technology Acceptance and Adoption by the Elderly: A Qualitative Study. In: Annual Research Conference of the South African Institute of Computer Scientists and Information Technologists, October 6-8 (2008), http://researchspace.csir.co.za/dspace/bitstream/10204/2514/ 1/Kotze_2008.pdf (Retrieved February 2, 2010)

3. Ziefle, M., Bay, S.: How to Overcome Disorientation in Mobile Phone Menus: A Comparison of Two Different Types of Navigation Aids. Human-Computer Interaction 21, 393-433 (2006)

4. Roring, R.W., Hines, F.G., Charness, N.: Age Differences in Identifying Words in Synthetic Speech. Human Factors: The Journal of the Human Factors and Ergonomics Society 49(7), 25-31 (2007)

5. Kausler, D.H., Salthouse, T.A., Saults, J.S.: Temporal memory over the adult lifespan. American Journal of Psychology 101, 207-215 (1988)

6. Howell, M., Love, S., Turner, M.: The impact of interface metaphor and context of use on the usability of a speech-based mobile city guide service. Behavior \& Information Technology 24(1), 67-78 (2005)

7. Howell, M., Love, S., Turner, M.: Visualization improves the usability of voice-operated mobile phone services. Human-Computer Studies 64, 754-769 (2006)

8. Logan, J.M., Sanders, A.L., Snyder, A.Z., Morris, J.C., Buckner, R.L.: Under-Recruitment and Non-Selective Recruitment. Neuron 33(5), 827-840 (2002)

9. Heim, A.W.: AH4 Group Test of Intelligence Manual. National Foundation for Educational Research, Slough (1967)

10. Baddeley, A.: Working Memory. University Press, Oxford (1986)

11. The British Psychological Society, Code of Ethics and Conduct: Guidance published by the Ethics Committee of the British Psychological Society. BPS, Leicester (2009) 